\title{
Evaluation des propriétés hydrauliques des aquifères fracturés des formations cristalline et métamorphique dans la région des Lacs (centre de la Côte d'Ivoire)
}

\author{
Assessment of the hydraulic properties of fractured aquifers \\ in crystalline and metamorphic formations in the region of Lacs \\ (Central Côte d'Ivoire)
}

\author{
G. Soro' ${ }^{1}$, N. Soro ${ }^{1}$, K.E. Ahoussi ${ }^{1}$, T. Lasm¹, F.K. Kouamé1 ${ }^{1}$, T.D. Soro' ${ }^{1}$, J. Biémi ${ }^{1}$
}

\begin{abstract}
RESUME
Dans la région des Lacs, centre de la Côte d'Ivoire (Afrique de l'Ouest), l'alimentation en eau potable des populations rurales est assurée en majorité par les eaux souterraines contenues dans les aquifères discontinus cristallin et cristallophyllien. L'objet de cet article est d'évaluer les propriétés hydrauliques de ces aquifères afin d'entreprendre des études pour la gestion optimale de cette ressource. Pour ce faire, une compilation de données regroupant les fiches techniques de forage de même que les essais de pompage disponibles dans la région a été faite. L'interprétation des essais de pompage par la méthode de remontée de Jacob a permis de déterminer 105 valeurs de transmissivité $(T)$ et de débit spécifique $(Q / S)$. Une analyse statistique des paramètres de forages et des paramètres hydrauliques déterminés a été réalisée. Les forages ont une profondeur totale qui varie de 49,50 à $99 \mathrm{~m}$ avec une moyenne de $69 \mathrm{~m}$. L'épaisseur des altérites est comprise entre 1,90 et $63,10 \mathrm{~m}$ pour une moyenne de $16,52 \mathrm{~m}$. Les forages ont un débit moyen de $2,32 \mathrm{~m}^{3} / \mathrm{s}$. Les profondeurs de venues d'eau sont en moyenne de 42,94 $\mathrm{m}$ dans la roche non altérée. Les transmissivités et les débits spécifiques s'échelonnent sur trois ordres de grandeur avec des coefficients de variation supérieurs à $100 \%$ traduisant ainsi l'hétérogénéité structurale du milieu aquifère. Ces deux paramètres sont également distribués selon la loi log-normale au seuil de $10 \%$. Une relation empirique significative entre la transmissivité et le débit spécifique a été établie. Elle permet d'estimer la transmissivité par l'expression $T=0,937(Q / S)^{1,118}$ avec $R^{2}=0,82$ dans un intervalle de confiance de $95 \%$ pour les aquifères de la zone d'étude. Ces résultats sont importants car, ils seront utiles dans les études de modélisation de ces aquifères et faciliteront la politique de gestion des eaux souterraines.
\end{abstract}

Mots clés: Région des Lacs, Côte d'Ivoire, Paramètres hydrauliques, statistiques, Aquifère fracturé.

\begin{abstract}
Groundwater is the main source of water supply for rural populations in the Region des Lacs area in central Côte d'Ivoire (West Africa). The area is underlain by the metamorphic and crystalline fractured hard rock aquifers. This paper focuses on the assessment of their hydraulic properties. To this end, a data base comprising pumping tests data and the technical reports were gathered. 105 values of transmissivity $(T)$ and specific capacity $(Q / s)$ have been deduced after pumping tests interpretation by the Jacob recovery method. Statistical analyses of all these data have been done. Depth of wells range from 49.50 to $99 \mathrm{~m}$ with an average of $69 \mathrm{~m}$. The thickness of the weathered zone has averaged $16.52 \mathrm{~m}$ and lies between 1.90 and $63.10 \mathrm{~m}$. Wells average rate is $2.32 \mathrm{~m}^{3} / \mathrm{s}$. The depths of open fractures are significantly in the first 60 meters of hard rock drilling and averaged $42.94 \mathrm{~m}$. The transmissivity and specific capacity of each aquifer span over several orders of magnitude revealing the strong heterogeneity of the aquifer. Both variables are lognormal variables A significant empirical relationship between $T$ and $Q / s$ was found
\end{abstract}

\footnotetext{
1 Laboratoire des Sciences et Technique de l'Eau et de l'Environnement (LSTEE), Université de Cocody, 22 B.P. 801 Abidjan 22, Côte d'Ivoire. Emails: marc_soro@yahoo.fr, soro_nagnin@yahoo.fr, ahoussi_kouassi@caramail.com, kouamef@yahoo.fr, paragafro@yahoo.fr, theophile_lasm@yahoo.fr, jbiemi@yahoo.fr
} 
$T=0,937(Q / s)^{1,118}$ with $R^{2}=0.82$. This relationship enabled the transmissivity data to be supplemented with the $95 \%$ prediction interval in order to assess the uncertainty associated with the estimates of transmissivity in the area of the study. These results are significant and can be used as an input in forthcoming modeling these aquifers and facilitate groundwater management policy.

Key words: Région des Lacs, Côte d'Ivoire, Hydraulic parameters, statistics, fractured aquifer.

\section{Introduction}

En Côte d'Ivoire, l'exploitation des ressources en eau souterraine a permis de faire face au problème de l'alimentation en eau potable des populations rurales grâce à l'investissement de nombreux capitaux (Fonds National de l'Hydraulique (FNH) de 1979 à 1980); 5è Fonds Européen pour le développement (FED), Banque Africaine de Développement (BAD), Banque Ouest Africaine de Développement (BOAD), Caisse Française de développement (CFD)/ $3^{\text {ème }}$ Conseil de l'Entente (CE3), etc. A la date du 31/01/2001, selon les sources de la Direction de l'Hydraulique Humaine (DHH), 19437 points d'eau ont été réalisés sur toute l'étendue du territoire (DHH, 2001). Ces ressources localisées dans les aquifères fracturés ou fissurés relativement bien protégés du fait de l'importante couverture en surface, sont de ce fait saines du point de vue bactériologique et parasitologique (Razack \& Lasm, 2006). Elles sont aussi excellentes du point de vue de la qualité chimique. Cet atout majeur a donc guidé les spécialistes des sciences de l'eau de l'Afrique de l'Ouest en général et de la Côte d'Ivoire en particulier à s'orienter vers ces aquifères fissurés ou fracturés. Mais l'exploitation de ces aquifères n'a été possible qu'avec l'avènement du marteau fond de trou dans les années 70 en Afrique de l'Ouest (Engalenc, 1978). Ces aquifères captés fournissent des débits dont la moyenne ne dépasse pas 3,2 $\mathrm{m}^{3} \mathrm{~h}^{-1}$ (Leblond, 1984). Ces débits servent donc à l'alimentation en eau potable des populations rurales dans le cadre des systèmes d'hydraulique villageoise (HV) ou d'hydraulique villageoise améliorée (HVA). L'objectif du programme national d'hydraulique villageoise (PNHV) lancé dans les années 70, était d'offrir 10 à $15 \mathrm{~L} /$ jour/habitant, soit un point d'eau pour 100 habitants. Par la suite ces besoins ont été revus à la hausse à partir des années 80 avec 15 à $20 \mathrm{~L} /$ jour/habitant (DHH, 2001). Aujourd'hui, pour satisfaire ces besoins, il faut multiplier ces chiffres par 4 voire 5 , soit au moins 40 à $100 \mathrm{~L} /$ jour/habitant (Soro, 2002). Un inventaire des différents ouvrages dans la région des Lacs a permis de dénombrer 1176 points d'eau (forages et puits modernes). Mais le constat est amer, car sur ces 1176 ouvrages, 538 sont abandonnés pour diverses raisons (panne mécanique, tarissement, qualité de l'eau, etc.) et 128 ont été déclarés négatifs car n'ayant pas atteint un débit minimum de $1 \mathrm{~m}^{3} / \mathrm{h}$ pour être déclaré positifs, ce qui fait un total de 766 ouvrages abandonnés, soit $65 \%$. Ce pourcentage élevé d'échec est alarmant et suscite beaucoup d'intérêts tant sur le plan scientifique qu'économique. Au plan scientifique, le mauvais choix des sites d'implantation des forages peut entraîner des dénoyages fréquents des nappes et être à l'origine des pannes mécaniques constatées au niveau des ouvrages. De même, la baisse des niveaux statiques des forages peut être attribuée aux déficits pluviométriques chroniques relevés en Côte d'Ivoire (Soro et al., 2007; Kouassi et al., 2007). Par ailleurs, les propriétés hydrauliques et géométriques des aquifères exploités ne sont connus que de façon sommaire (Leblond, 1984; N'guessan, 1985). Au plan économique, le nombre d'ouvrages à réaliser dans la zone s'élève à 203 auxquels il faut ajouter les forages à restaurer. Tout ceci nécessite l'investissement d'une masse financière importante de la part de l'Etat ivoirien et de ses partenaires au développement. En outre la région des Lacs présente un taux de satisfaction des besoins en eau inferieur à 50\% (DHH, 2001). A toute cette kyrielle de problèmes énumérés, il faut enfin tenir compte de l'impact à long terme sur la demande en eau et de l'effet du transfert effectif de la capitale politique à Yamoussoukro. De ce fait, le développement d'une politique efficace de gestion des ressources de ces aquifères nécessite donc la connaissance de la géométrie et des propriétés de ces réservoirs aquifères. C'est pour répondre à cette problématique que la présente étude tire son essence. Elle a pour objectif principal d'évaluer les propriétés des paramètres hydrauliques des aquifères fracturés des formations cristalline et métamorphique dans la région des Lacs. Il s'agit d'une part, d'analyser les paramètres qui ont une influence sur la géométrie des aquifères et, d'autre part, de concevoir une relation empirique entre la transmissivité et le débit spécifique. Ce faisant, on pourra estimer les transmissivités où sont 
rares et cela dans l'optique d'une modélisation future de ces aquifères pour une gestion optimale de leur ressource.

\section{Contexte général de la zone d'étude}

\section{Situation géographique}

Le secteur d'intérêt se situe dans la région des Lacs, centre de la Côte d'Ivoire, entre $6^{\circ} 30$ et $7^{\circ} 35$ de latitude nord et $4^{\circ} 40$ et $5^{\circ} 40$ de longitude ouest (fig. 1). Au plan administratif, la région des Lacs a pour principale ville, Yamoussoukro, capitale politique du pays. Elle couvre une superficie d'environ $6000 \mathrm{~km}^{2}$ pour une population estimée en 2001 à 477156 habitants (INS, 2001). L'activité principale est l'agriculture. La zone est drainée par le fleuve Bandama et les affluents du N'zi et est dotée de nombreuses retenues d'eau destinées à l'agriculture.

\section{Géologie}

La région des Lacs appartient au domaine protérozoïque de la dorsale de LEO (fig. 1). Elle est située dans le birimien de la Côte d'Ivoire qui appartient au domaine «Baoulé-Mossi» du craton ouest africain. Les principales formations géologiques sont constituées de roches magmatiques et métamorphiques (Yacé, 1976, 2002; Leblond, 1984; N'guessan, 1985). Les roches magmatiques appartiennent au complexe éburnéen et comprennent les granitoïdes éburnéens. Ce sont des granites à biotite, des granites à deux micas, des migmatites, des granodiorites concordants et des pegmatites. Les formations volcano-sédimentaires sont représentées par les schistes (vert et ardoisier), les quartzites, les métasédiments indifférenciés et les roches vertes du birimien. Les roches vertes sont des roches basiques dont la nature originelle est encore décelable et des roches complètement transformées correspondant aux faciès amphibolite et schiste vert (Soule de Lafont, 1956). Ce sont des schistoïdes caractérisées par une légère schistosité et de structure granoblastique. Elles se fissurent mais ne se délitent pas et se comportent comme des schistes (Engalenc, 1978). La couleur verte à l'affleurement leur a conféré ce nom générique de roches vertes. Ainsi sous ce vocable, on y trouve des métabasaltes, des métaandésites, métadolérites et des métagabbros (Yacé, 1967, 2002). Sur le terrain, elles se présentent sous forme allongées, étroites avec un relief défavorable à l'implantation de sites de villages (N'guessan, 1985). Ces deux principales formations géologiques conduisent à la formation d'un contexte hydrogéologique caractérisé par deux types d'aquifères; un aquifère d'altérites et un aquifère fissuré ou fracturé.

\section{Hydrogéologie}

L'aquifère d'altérites a une porosité totale d'interstices élevée mais une faible porosité efficace et une très faible perméabilité (Leblond, 1984). Cet aquifère a fait l'objet de beaucoup de sollicitations en vue de répondre aux problèmes d'eau en Afrique de l'ouest. Mais la teneur en minéraux argileux élevée de ces altérites donne une eau de qualité médiocre et des débits très peu élevés de $1,3 \mathrm{~m}^{3} / \mathrm{h}$. Cependant, jusqu'en 1975, ces altérites lorsqu'elles sont saturées en eau furent exploitées à l'aide de puits modernes à grand diamètre avec des profondeurs ne dépassant pas les 30 mètres et équipés de pompe à motricité humaine pour l'alimentation en eau des villages. Ces mêmes altérites sont captées par les paysans à l'aide d'ouvrages appelés puits paysans ou puisards. La puissance de la zone d'altération est variable. Elle peut atteindre en zone humide de la Côte d'Ivoire une épaisseur de plus de $50 \mathrm{~m}$ sur roches basiques ou schistes (Fahy, 1980, 1981). Sur roches acides, elle atteint une trentaine de mètres au maximum. Dans les zones fracturées, elle descend jusqu'à 70 à 90 m (Engalenc, 1978). Mais l'épaisseur maximum moyenne est de 40 à $50 \mathrm{~m}$ quel que soit le pays considéré ou le climat ce qui suggère que la profondeur de l'altération est sous la dépendance directe des fractures ouvertes (Engalenc, 1978). Ces aquifères contiennent généralement des nappes libres qui reçoivent directement les précipitations. Les niveaux piézométriques fluctuent entre la saison des pluies et la saison sèche. La baisse du niveau piézométrique est souvent due au drainage vers les cours d'eau pérennes ou autres points d'eau environnants tels que les barrages, puits, et forages (Marechal et al., 2006). Contrairement aux altérites, le socle a une porosité matricielle pratiquement nulle sauf dans la zone superficielle correspondant à la limite inférieure des arènes grenues; la perméabilité est bonne notamment dans les zones à forte densité de fracturation. Il s'agit de perméabilité de fissure et de fracture. La transmissivité est très inégale d'un point à un autre du fait des variations très rapides de l'épaisseur de la zone fracturée. Cette 


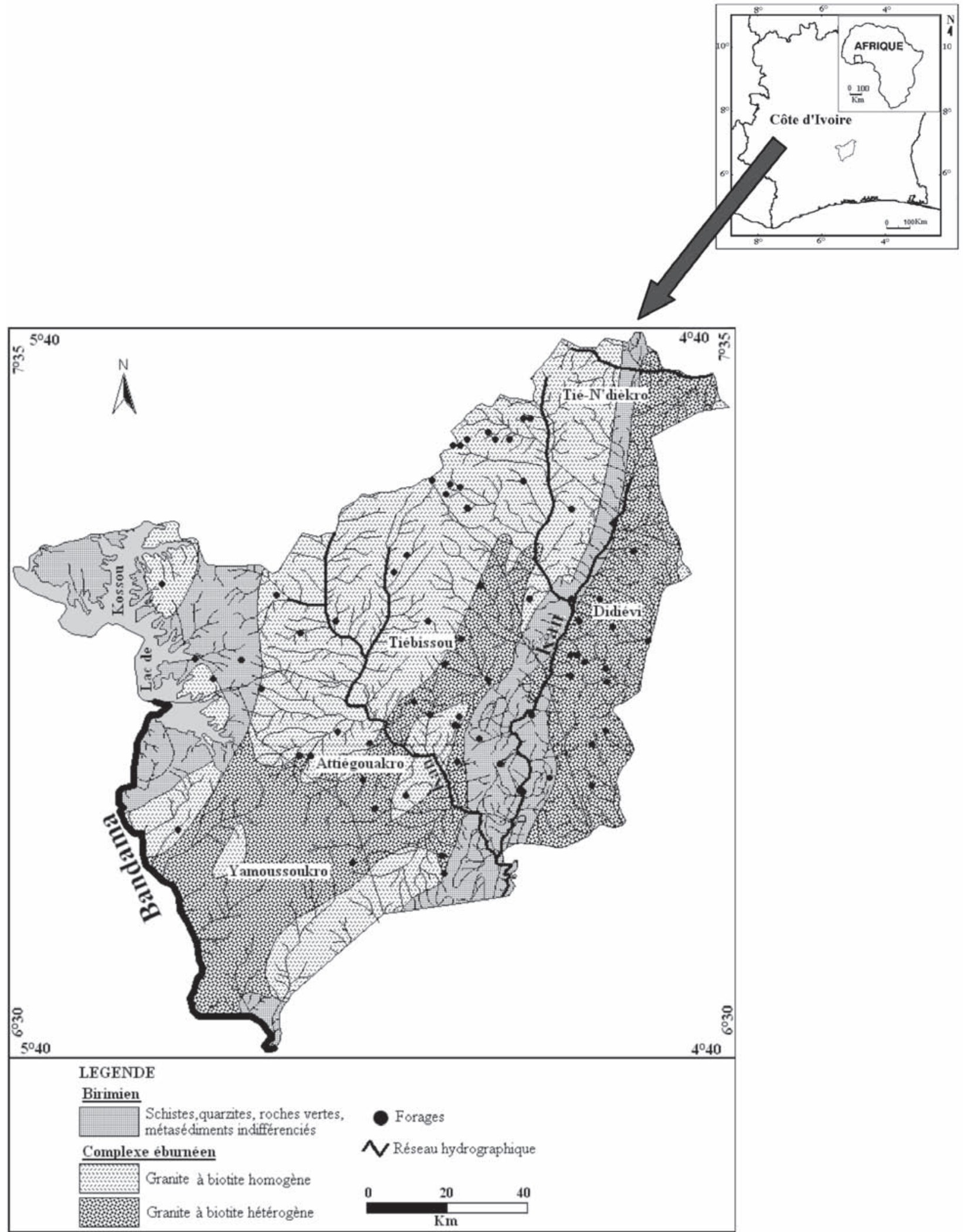

Fig. 1.-Situation géographique et géologie de la zone d'étude. 
variabilité spatiale des propriétés hydrodynamiques de ces roches donne aux aquifères qu'elles renferment le terme d'aquifère «discontinus».

L'hydrogéologie des milieux fissurés ou discontinus a connu durant ces dernières années un grand essor grâce à l'utilisation de nouveaux matériels de prospection. Cet aquifère est exploité par des forages dont la profondeur atteint très rarement les $100 \mathrm{~m}$ (Detay et al., 1989). Il est beaucoup sollicité pour ces eaux dotées d'une bonne qualité sanitaire. Mais la productivité des ouvrages hydrauliques varie de manière aléatoire. Compte tenu de leurs perméabilité et porosité relativement faibles, la productivité des aquifères de socle est modeste. Les forages sont susceptibles de produire des débits exploitables compris entre $2-3$ et 10 à $20 \mathrm{~m}^{3} / \mathrm{h}$ (Lachassagne \& Wyns, 2005). Cette productivité est influencée par le faciès lithologique, la présence ou non d'altérites, la connectivité hydraulique des fractures recoupées par les ouvrages (M'bonu, 1991). En milieu cristallin et cristallophyllien, on note au sein de chaque aquifère deux niveaux: un premier à fonction capacitive et un second à fonction conductrice. Le modèle conceptuel d'aquifère de socle généralement admis est constitué d'un réservoir d'altérites semi-perméable, à fonction capacitive et alimenté par la surface. Il surmonte un aquifère de fissures ou de failles, captif, drainant la couverture, à fonction essentiellement conductrice (Engalenc, 1978; Detay et al., 1989). En Afrique de l'Ouest, dans les aquifères de fissure, l'importance des débits au niveau des granitoïdes est liée à la densité de fissuration et à la perméabilité de la tranche altérée (Savadogo, 1984; Biémi, 1992). Dans les schistes, une schistosité développée peut augmenter la porosité. De même la présence de filons de quartz dans ces roches constitue des lieux privilégiés de circulation des eaux souterraines. La transimissivité équivalente de cet aquifère est comprise entre $10^{-4}$ et $10^{-3} \mathrm{~m}^{2} \mathrm{~s}^{-1}$ (Engalenc, 1978; Faillat, 1986). Sur le site expérimental de Yamoussoukro dans les granitoïdes éburnéens, un coefficient d'emmagasinement compris entre $10^{-4}$ et $10^{-3}$ a été obtenu (Faillat, 1986). Lors de pompages, les fissures drainent les altérites saturées. Le schéma de fonctionnement des nappes de fissures est classique et utilisé pour les autres milieux. Ainsi, on a une zone d'alimentation, directe sur ou au contact d'affleurements ou indirecte (drainance) sous les altérites saturées, une zone de circulation plus ou moins développée et enfin, une zone d'émergence directement à partir d'affleurements et le plus souvent par l'intermédiaire des nappes d'altérites (Faillat, 1987). Les nappes de ces aquifères sont semi-captives dans la majeure partie et très rarement captives sous les altérites. Ces dernières années un nouveau concept est en vigueur sur le fonctionnement des aquifères de socle. Cette schématisation des aquifères de socle a été proposée par Wyns et al. (1999); Cho et al. (2003); Marechal et al. (2004); Lachassagne \& al. (2005). Sous les altérites, on trouve un «horizon fissuré», stratiforme, de 50 à $100 \mathrm{~m}$ d'épaisseur, auquel l'aquifère de socle doit sa perméabilité (Cho et al., 2003). La zone fissurée est constituée par une densité de fractures horizontales dans la roche saine qui décroît avec la profondeur. Elle est caractérisée par la présence de fissures subhorizontales et subverticales (Lachassagne \& al., 2001).

\section{Hydrostructurale}

La tectonique de la Côte d'Ivoire est dominée par l'existence de grands accidents ductiles N-S senestre (Fabre, 1985). Ces accidents sont qualifiés de décrochements de grandes amplitudes, postérieurs à l'orogénèse éburnéenne. Les accidents orientés $\mathrm{N} 170^{\circ}$ à $\mathrm{N} 190^{\circ}$ ont influencé le tracé du réseau hydrographique des grands fleuves de la Côte d'Ivoire parmi lesquels nous pouvons citer: le Bandama, le Sassandra, le N'zi, la Comoé. Une deuxième direction $\mathrm{N} 30^{\circ}$ à $\mathrm{N} 40^{\circ}$ est représentée par les accidents des Monts Trou, par l'accident de Soubré (sud-ouest de la Côte d'Ivoire) et se poursuit dans le Yaouré (centre de la Côte d'Ivoire) jusqu'à la faille du HautN'zi, et par celui de Dimbokro sur le Bas-N'zi. Une troisième famille d'accidents d'orientation $\mathrm{N} 120^{\circ}$ à $\mathrm{N} 130^{\circ}$ parallèle aux filons doléritiques du sud-ouest et qui auraient été engendrés par un raccourcissement NNE-SSW au cours de l'orogénèse Panafricaine (200MA) (Biémi, 1992). Enfin, on note une quatrième famille d'accidents de direction $\mathrm{N} 80^{\circ}-\mathrm{N} 100^{\circ}$. Dans le centre de la Côte d'Ivoire, où se situe la région des Lacs, on note la présence de trois grandes failles: la faille de Soubré-Kossou (NE-SW), la faille du N'zi (N-S) et la faille Dimbokro-Bassa (SW-NE). La première et la troisième sont parallèles aux structures alors que la seconde les recoupe (N'guessan, 1985). Dans la région des Lacs, les travaux de Yao (1998) ont décrit des linéaments de directions N00 à N10, N020 à N030, N040 à N050 et N130 à N150. La direction N130 à N150 qui représente les ensembles granitiques est dominante. Par contre, la direction N020 à N030 est toujours bien représentée et est caractéristique des formations 
volcano-sédimentaires du birimien. Dans ce bassin, les couches sont orientées selon une direction N4050, dite direction Baoulé (Fabre, 1985). Dans la même zone, une étude de la fracturation dans le bassin du Yaouré au centre de la Côte d'Ivoire a montré la prédominance des familles de fractures $\mathrm{N} 75-80^{\circ}$ et N155-120 . L'examen de nombreux affleurements a permis de déceler des fractures conjuguées, des fractures avec stries, des fentes de tension qui montrent l'existence d'au moins trois directions de raccourcissement NNE-SSW, E-W et NW-SE depuis la fin de l'Eburnéen (Faillat, 1986). Si la succession des phases tectoniques responsables de ces déformations n'est pas élucidée, il apparait cependant clairement que les événements tectoniques observés et datés sur les marges du Craton Ouest africain aient créé de nouvelles fractures ou réactivé les anciennes (Faillat, 1986). De ce fait, les réseaux de fractures ont donc pu être maintenus hydrauliquement actifs grâce à la persistance des mouvements tectoniques compressifs ou distensifs jusqu'à une époque récente. Au niveau hydrostructurale, ces accidents jouent un rôle très important dans la mise en place des aquifères de fractures ou de fissures.

\section{Méthodologie}

\section{Données et matériels}

Les données utilisées dans ce travail proviennent de la campagne de forages du projet Caisse Française de développement/3 ${ }^{\text {ème }}$ phase du Conseil de l'entente (CFD/CE3) réalisé en 1998 dans la région des Lacs. Elles ont été fournies par l'antenne de la Direction Territoriale de l'Hydraulique de Yamoussoukro. Ces données sont constituées de fiches techniques des forages et des données des essais de pompage. Dans le cadre de cette étude, un total de 105 fiches techniques de forages a été retenu du fait de la qualité de leurs données. Tous les forages ont des diamètres compris entre $6 " 1 / 2$ et $9 " 1 / 4$. Ils sont équipés sur toute la profondeur forée avec des PVC de diamètre 5". Les crépines sont placées au droit des arrivées d'eau. Les profondeurs des forages retenus dans cette étude sont comprises entre 50 et 100 m. Dans cette étude, les forages ont une pénétration quasi-totale dans l'aquifère. Après le développement du puits, les pompages d'essai sont exécutés par paliers enchaînés d'une durée de 4 à 12 heures avec généralement trois paliers. La remontée est suivie pendant une heure d'observation. Les mesures de rabattement de la nappe ont été effectuées directement dans le puits de pompage en l'absence de piézomètre d'observation. Tous les essais de pompage ainsi que les mesures de remontée ont été assemblés et analysés. Les essais de pompage de moins de 12 heures ont été éliminés.

\section{Détermination de la transmissivité en utilisant les courbes de remontée des essais de pompage}

L'interprétation de ces essais de pompage repose sur les expressions hydrodynamiques en régime transitoire de Theis (1935) et de Jacob (1947). Cette interprétation a pour objet de déterminer les paramètres hydrauliques des aquifères notamment la transmissivité. En général, ces méthodes s'appliquent pour les pompages de longue durée, mais à partir des essais de courte durée, à condition d'atteindre un palier au cours de chaque cycle, l'on peut calculer ces paramètres (Lasm, 2000). L'absence de piézomètres d'observation ne permet pas de déterminer le coefficient d'emmagasinement (CEFIGRE, 1990, Detay, 1993).

Le puits de pompage étant le puits d'observation il est recommandé dans ces conditions d'utiliser les données de la remontée pour les interprétations (Razack \& Lasm, 2006). En effet, lors de la descente, le plan d'eau est perturbé au niveau des crépines créant ainsi une perte de charge quadratique qui biaise les résultats (Detay, 1993; De Marsily, 1994; Lasm, 2000; Razack \& Lasm, 2006). Au cours de la descente, le niveau dynamique dans le puits représente mal la charge dans la nappe au voisinage du puits. Les valeurs de transmissivité obtenues sont dans ce cas surestimées ou sous-estimées. Par contre, à la remontée, tous ces phénomènes sont annulés, et l'on observe véritablement le niveau de la nappe dans le puits, ce qui permet une bonne interprétation. L'intérêt des courbes de remontée est très grand pour les essais de débits. Selon Detay (1993), l'analyse des courbes de remontée donne des résultats aussi sûrs, sinon meilleurs que ceux tirés de l'observation de la descente. Il existe deux méthodes d'interprétation des courbes de remontée d'un essai de pompage (De Marsily, 1994):

- la méthode Houpert-Pouchan;

- la méthode de Jacob: courbe de remontée pour les nappes captives.

Les nappes étudiées sont prises comme étant captives et de ce fait, on suppose qu'elles répondent 
aux hypothèses de la méthode de Jacob. C'est pourquoi, cette méthode a été utilisée pour estimer les transmissivités. Elle repose sur l'application du principe de superposition. Pour simuler l'arrêt du pompage, on ajoute au débit $Q$ extrait, une injection de même débit constant $(-Q)$. Le rabattement $S_{r}$ est donc la somme des rabattements $\left(s+s^{\prime}\right)$ et est appelé rabattement résiduel (équation 1).

$$
S_{r}=\frac{0,183}{T} Q\left(\log \frac{2,25 T\left(t_{p}+t_{r}\right)}{r^{2} S}-\log \frac{2,25 T t_{r}}{r^{2} S}\right)
$$

Avec

$S_{r}:$ rabattement résiduel.

$t_{p}$ : la durée du pompage (s) jusqu'à l'arrêt de la pompe

$t_{r}$ : le temps écoulé depuis l'arrêt du pompage (s'): temps de remontée.

En simplifiant l'équation (1), on obtient l'équation (2) suivante

$$
S_{r}=\frac{0,183 Q}{T} \log \left(1+\frac{t_{p}}{t_{r}}\right)
$$

Dans cette équation, l'expression $\left(t_{p}+t_{r}\right)$ est remplacée par la moyenne logarithmique pondérée du temps $\bar{t}$ (temps corrigé). Ce temps corrigé $\bar{t}$ représente le temps auquel se serait produit le rabattement dans un certain piézomètre si le débit pompé était resté constant depuis le début et égal au débit réel au temps $t$ (Kruserman et al., 1974; Kruserman et De Ridder, 1991; Chapuis, 2007). On trace ensuite la courbe $\mathrm{Sr}$ en fonction de $\log \left(\bar{t} / t_{r}\right)$.

La pente de la droite obtenue correspond à la variation de $\mathrm{Sr}$ par cycle logarithmique $\Delta \mathrm{Sr}$ et permet de déduire $\mathrm{T}$ par la formule (3) suivante:

$$
T=\frac{0,183 Q}{\Delta s_{r}}
$$

Le débit spécifique a été évalué à la fin du troisième palier après 12 heures de pompage. Les rabattements n'ont pas été corrigés des pertes de charges avant la détermination du débit spécifique.

\section{Estimation de la transmissivité à partir du débit spécifique à travers une relation empirique}

La transmissivité et la perméabilité sont les paramètres les mieux indiqués pour la caractérisation des propriétés géométriques d'un aquifère. Les paramètres hydrauliques des aquifères fracturés sont souvent sous le contrôle de la géométrie des fractures (Hamm et al., 2005). Le débit spécifique est l'un des paramètres hydrauliques dont la mesure est facile sur un ouvrage, il est le rapport du débit de pompage sur le rabattement observé dans le puits. De ce fait, ces valeurs sont généralement plus abondantes dans les archives hydrogéologiques que les valeurs de transmissivité ou de perméabilité. C'est pourquoi, des méthodes simples et moins chères sont souvent préférées pour déterminer ces paramètres. L'une de ces méthodes consiste à estimer la transmissivité à travers une relation qui lie cette dernière au débit spécifique. Pour justifier la détermination de la transmissivité sur la base des données de débits spécifiques, il est nécessaire de comprendre que la transmissivité est linéairement proportionnelle au débit spécifique d'un puits selon l'équation de Dupuit-Thiem pour les aquifères non confinés et pour les aquifères confinés selon l'équation de Theis (Hamm et al., 2005). Une telle approche est basée sur l'équation (4) d'équilibre de Thiem pour les aquifères confinés (Rotzoll \& ElKadi, 2008):

$$
T=\frac{2,3 Q}{2 \pi s} \ln \left(\frac{r_{2}}{r}\right)
$$

Où $T$ est la transmissivité $\left(\mathrm{m}^{2} / \mathrm{s}\right)$.

$Q$ le débit du puits $\left(\mathrm{m}^{3} / \mathrm{s}\right)$.

$S$ le niveau du rabattement dans le puits de pompage $(\mathrm{m})$.

$r_{2}$ : le rayon d'influence $(\mathrm{m}), r$ le rayon du puits de pompage $(\mathrm{m})$.

L'équation de Thiem peut être appliquée aux aquifères non confinés quand le rabattement est insignifiant comparé à l'épaisseur de l'aquifère (Gingerich, 1999). Thomasson et al. (1960) in Rotzoll \& El-Kadi (2008) ont simplifié l'équation (4) en utilisant des valeurs théoriques pour le terme logarithmique. Ainsi, en combinant une valeur moyenne du terme logarithmique avec les autres constantes, la relation entre $T$ et $Q / s$ est réduite à l'équation (5) suivante:

$$
T=A_{1} \cdot Q / s
$$

Où $Q / s$ est le débit spécifique $\left(\mathrm{m}^{2} / \mathrm{s}\right)$, la constante $A_{1}$ sans dimension est comprise entre 0,9 et 1,52, avec une moyenne de 1,18. 
Diverses études ont décrit des relations empiriques entre $T$ et $Q / s$ pour les aquifères fracturés (Razack \& Huntel, (1991), Acheampong \& Hess, 1998; Jalludin \& Razack, 2004; Hamm et al., 2005; Razack \& Lasm, 2006; Yidana et al., 2007). Très souvent la corrélation est meilleure entre les valeurs logarithmiques de $T$ et $Q / s$; et une relation linéaire peut être écrite selon l'équation (6) suivante:

$$
T=A_{2}(Q / s)^{\mathrm{D}}
$$

Où $A_{2}$ et $D$ sont les coefficients de régression de la relation puissance.

Utilisant la même méthode, différentes relations empiriques entre $T$ et $Q / s$ ont été proposées dans les aquifères fracturés d'Afrique et ailleurs. Ainsi, dans les plaines de l'Afram dans le Sud du bassin de la Volta au Ghana, Acheampong \& Hess (1998) en utilisant 28 paires de données de transmissivité et de débit spécifique ont trouvé $T=20(Q / s)^{1,02}\left(R^{2}=0,69\right)$. Récemment dans la même région, Yidana et al. (2007) ont obtenu $T=0,769(Q / s)^{1,075}\left(R^{2}=0,83\right)$. Jalludin \& Razack (2004) ont trouvé $T=2,99(Q / s)_{\text {cor }}{ }^{0,938}$ $\left(R^{2}=0,82\right)$, dans les aquifères volcaniques de la République de Djibouti, où $(Q / s)$ cor est le débit spécifique corrigé. Dans les aquifères cristallin et métamorphique fracturés de la région de ManDanané, à l'ouest de la Côte d'Ivoire, Razack \& Lasm (2006) proposent pour 118 paires ( $T$ et $Q / s$ ) $T=0,89(Q / s)^{1,30}\left(R^{2}=0,88\right)$. Hamm et al. $(2005)$ dans les aquifères volcaniques de l'île de Jeju en Corée du Sud obtiennent $T=0,99(Q / s)^{0,89}\left(R^{2}=0,94\right)$. Ces différentes relations empiriques varient pour différents paramètres hydrogéologiques donnés. On constate également que ces expressions sont aussi différentes dans des environnements géologiques similaires. Toute chose qui indique qu'elles sont réellement spécifiques au site d'étude (Razack \& Lasm, 2006). De même, ces relations dépendent des méthodes utilisées pour déterminer $T$ et $Q / s$. En se basant sur cette donne, une relation empirique entre la transmissivité et le débit spécifique a été proposée pour les aquifères discontinus de la région des Lacs. Cette relation basée sur la méthode de régression des moindres carrés permet de reproduire un champ de transmissivités avec des erreurs minimales. Les valeurs de débit spécifique $(Q / s)$ et de transmissivité $(T)$ sont reportées dans un diagramme bi-logarithmique. Si le nuage des points résultant s'aligne suivant une droite, l'équation de celleci est déterminée ainsi que son coefficient de détermination qui traduit la qualité de l'ajustement. Cette relation est souvent très significative lorsque les valeurs de $T$ et de $Q / s$ s'étendent sur plusieurs ordres de grandeurs. Razack \& Lasm (2006) estiment qu'une correction du débit spécifique des pertes de charges dues à la turbulence de l'écoulement, pourrait améliorer la relation empirique entre $T$ et $Q / s$. En se basant sur cette équation, une relation empirique entre la transmissivité et le débit spécifique a été proposée pour les aquifères discontinus de la région des Lacs.

\section{Résultats}

\section{Analyse statistique des paramètres des forages}

Les paramètres statistiques qui caractérisent les 105 forages étudiés ont été reportés dans le tableau 1. La profondeur totale de forage varie de 49,50 à $99 \mathrm{~m}$ avec une moyenne de $69 \mathrm{~m}$. Cette profondeur est souvent fixée par les études géophysiques de bureau. Mais lors de l'exécution de l'ouvrage, elle est sujette à l'obtention d'un débit appelé débit positif et fixé lors du démarrage du projet. L'épaisseur des altérites est comprise entre 1,90 et $63,10 \mathrm{~m}$ avec une moyenne de 16,52 m. On dénombre souvent jusqu'à quatre arrivées d'eau qui correspondent aux profondeurs hydrauliquement actives. Mais, ce sont les premières arrivées d'eau qui sont les plus productives. La profondeur moyenne des arrivées d'eau est de 42,94 m avec des extrêmes allant de 10 et $87 \mathrm{~m}$. Le niveau statique dans les forages varie de 3,5 à $32,30 \mathrm{~m}$ avec une moyenne de 13,80 m. Le débit des forages est compris entre 0,10 et $10,80 \mathrm{~m}^{3} / \mathrm{s}$ pour une moyenne de $2,32 \mathrm{~m}^{3} / \mathrm{s}$. Il est souvent déterminé en fonction des objectifs de la campagne. Les différents coefficients de variation déterminés sont inférieurs à 100 en dehors de celui du débit des forages qui est légèrement supérieur à 100. Ces faibles valeurs traduisent une faible dispersion des paramètres étudiés. Elles montrent également qu'il existe une certaine homogénéité au niveau de ces valeurs.

Les résultats de l'analyse statistique de $T$ et de $Q / s$ sont consignés dans le tableau 1 . Les valeurs de transmissivité sont comprises entre $1,15 \cdot 10^{-6}$ et $4,48 \cdot 10^{-4} \mathrm{~m}^{2} / \mathrm{s}$ soit sur trois ordres de grandeur, avec une moyenne de $4,95 \cdot 10^{-5} \mathrm{~m}^{2} / \mathrm{s}$. Le débit spécifique est utilisé pour montrer la productivité d'un puits. En régime d'écoulement permanent, le débit spécifique est une fonction du rayon du puits, du degré de pénétration dans l'aquifère et de la transmissivité. 
Tableau 1.-Statistiques élémentaires des paramètres des forages

\begin{tabular}{lccccc}
\hline Paramètres & Minimum & Maximum & Moyenne & Ecart type & CV \\
\hline$P t(\mathrm{~m})$ & 49,50 & 99,00 & 69,25 & 13,77 & 19,88 \\
Epalt $(\mathrm{m})$ & 1,90 & 63,10 & 16,52 & 13,22 & 80,07 \\
$N S(\mathrm{~m})$ & 3,55 & 32,30 & 13,80 & 6,76 & 48,97 \\
$A E 1(\mathrm{~m})$ & 10,00 & 87,00 & 42,94 & 17,64 & 41,08 \\
$Q\left(\mathrm{~m}^{3} / \mathrm{s}\right)$ & 0,10 & 10,80 & 2,32 & 2,45 & 105,70 \\
$T\left(\mathrm{~m}^{2} / \mathrm{s}\right)$ & $1,15 \cdot 10^{-6}$ & $4,48 \cdot 10^{-4}$ & $4,95 \cdot 10^{-5}$ & $8,58 \cdot 10^{-5}$ & 173 \\
$Q / s\left(\mathrm{~m}^{2} / \mathrm{s}\right)$ & $2,30 \cdot 10^{-6}$ & $5,63 \cdot 10^{-4}$ & $5,19 \cdot 10^{-5}$ & $7,07 \cdot 10^{-5}$ & 136 \\
\hline
\end{tabular}

Pt: Profondeur totale.

Epalt: Epaisseur d'altération.

NS: Niveau statique.

$A E 1$ : première arrivée d'eau.

$Q$ : débit du forage.

$T$ : Transmissivité.

$Q / s$ : Débit spécifique.

En régime transitoire, le débit spécifique est une fonction du temps, du coefficient d'emmagasinement, du puits tout comme du rayon de ce dernier, du degré de pénétration dans l'aquifère et de la transmissivité. Les valeurs de débit spécifique estimées varient entre $2,30 \cdot 10^{-6}$ et $5,63 \cdot 10^{-4} \mathrm{~m}^{2} / \mathrm{s} / \mathrm{m}$ avec une moyenne de $5,19 \cdot 10^{-5} \mathrm{~m}^{2} / \mathrm{s} / \mathrm{m}$. Tout comme les transmissivités, les débits spécifiques s'étendent sur trois ordres de grandeur. Le coefficient de variation $(\mathrm{CV})$ qui représente le rapport de l'écart-type sur la moyenne traduit la dispersion au niveau d'un échantillon. Les valeurs des différents CV sont élevées et dépassent $100 \%$. Elles confirment ainsi la forte dispersion de la transmissivité et du débit spécifique. Cette dispersion reflète l'hétérogénéité structurale du milieu géologique et l'effet de modification de l'aquifère par l'homme lors de l'exécution du forage. Les transmissivités ont été subdivisées en trois classes (tableau 2). La classe la plus importante est celle de la moyenne qui a des valeurs qui oscillent entre $10^{-5}$ et $10^{-4} \mathrm{~m}^{2} / \mathrm{s}$ avec un effectif de 51 . Elle représente $48,6 \%$ de l'effectif total. Elle est suivie en terme d'effectif par la classe faible avec un effectif de 40 valeurs soit $38,1 \%$ du total. Le pourcentage cumulé de la classe moyenne avec celui de la classe forte donne $51 \%$ de l'effectif total. De ce fait, plus de la moitié des ouvrages ont des transi-

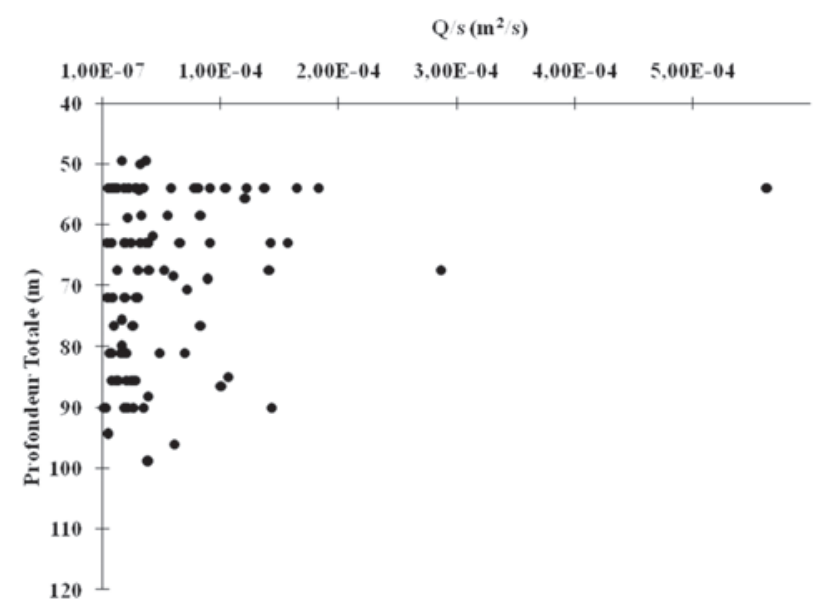

Fig. 2.-Profondeur totale des forages en fonction du débit spécifique.

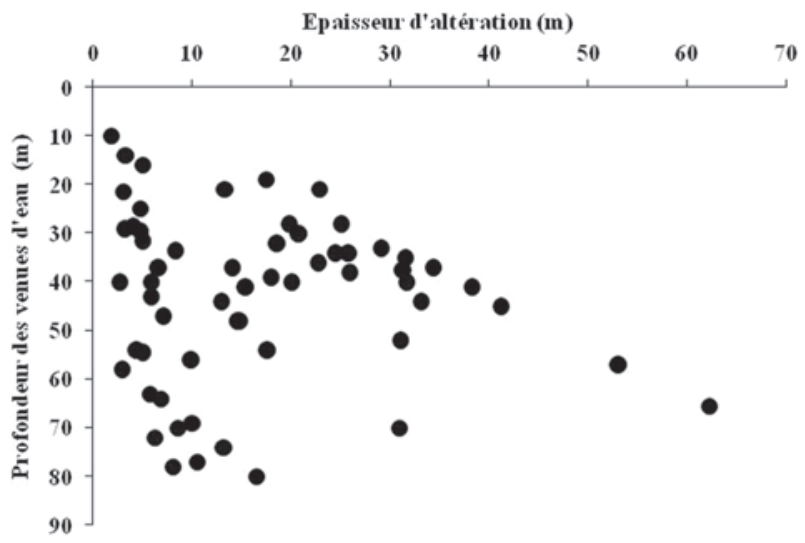

Fig. 3.-Distribution des profondeurs des venues d'eau en fonction des épaisseurs d'altération.

missivités supérieures à $10^{-5} \mathrm{~m}^{2} / \mathrm{s}$. Les figures 2 et 3 représentent respectivement les graphes de la profondeur totale des forages en fonction du débit spécifique et de la profondeur des venues d'eaux (profondeur hydrauliquement active) en fonction l'épaisseur d'altération. L'analyse du graphe de la figure 2 montre l'absence de corrélation significative entre ces deux paramètres. Par contre, les débits spécifiques les plus élevés correspondent aux forages les moins profonds. La figure 3 montre que les profon-

Tableau 2.-Classe des transmissivités dans la région des Lacs

\begin{tabular}{lccccc}
\hline Classe des transmissivité & Effectif & Minimum & Maximum & Moyenne & Ecart-type \\
\hline $10^{-6} \leq T \leq 10^{-5}\left(\mathrm{~m}^{2} / \mathrm{s}\right)$ & 40 & $1,15 \cdot 10^{-6}$ & $9,70 \cdot 10^{-6}$ & $5,08 \cdot 10^{-6}$ & $2,84 \cdot 10^{-6}$ \\
$10^{-5} \leq T \leq 10^{-4}\left(\mathrm{~m}^{2} / \mathrm{s}\right)$ & 51 & $1,04 \cdot 10^{-5}$ & $9,73 \cdot 10^{-5}$ & $3,45 \cdot 10^{-5}$ & $2,51 \cdot 10^{-5}$ \\
$T>10^{-4}\left(\mathrm{~m}^{2} / \mathrm{s}\right)$ & 14 & $1,02 \cdot 10^{-4}$ & $4,48 \cdot 10^{-4}$ & $2,31 \cdot 10^{-4}$ & $1,18 \cdot 10^{-4}$ \\
\hline
\end{tabular}




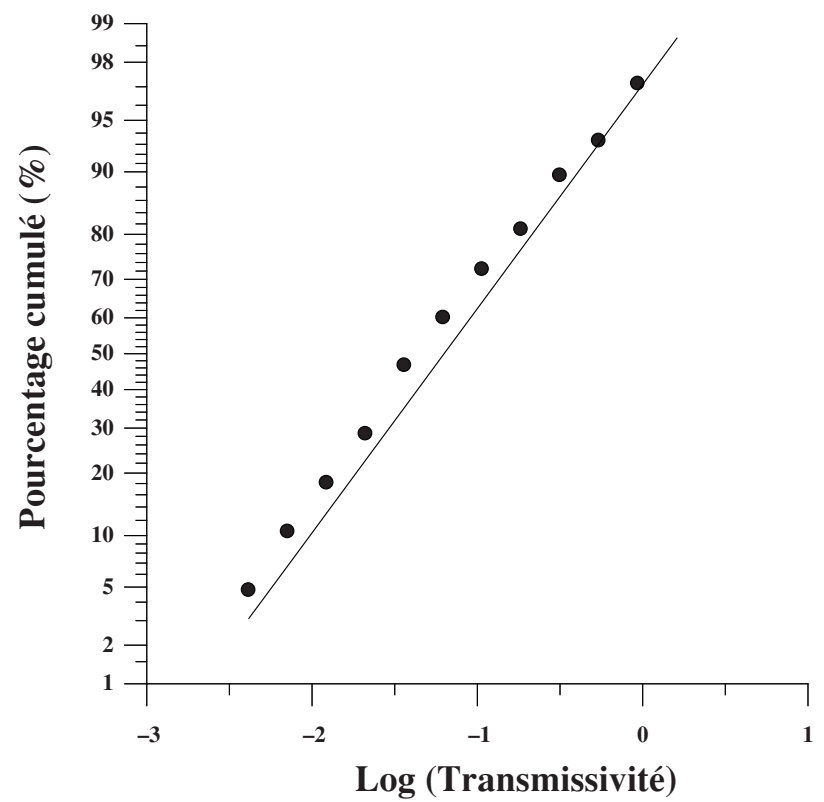

Fig. 4.-Fréquence de distribution de la transmissivité $\left(\mathrm{m}^{2} / \mathrm{h}\right)$ dans un diagramme lognormal.

Tableau 3.-Résultats du test de Khi deux

\begin{tabular}{llccc}
\hline \multirow{2}{*}{ Loi de distribution } & Paramètres & $\mathrm{c}^{2}$ calculé & $\begin{array}{c}\mathrm{c}^{2} \text { théorique } \\
(a=10 \%)\end{array}$ & $\begin{array}{c}\text { Degré } \\
\text { de liberté }\end{array}$ \\
\hline Loi lognormale & $\log T$ & 3,24 & 12 & 7 \\
\cline { 2 - 5 } & $\log (Q / s)$ & 2,43 & 2,71 & 1 \\
\hline
\end{tabular}

deurs hydrauliquement actives sont importantes pour une épaisseur d'altération comprise entre 0 et $30 \mathrm{~m}$. De même, le maximum des venues d'eau se rencontre dans les 60 premiers mètres forés dans la roche non altérée. Les valeurs brutes de $T$ et $Q / s$ ont été transformées en $\log$. La distribution des fréquences des valeurs transformées $(\log T$ et $\log Q / s)$ dans un diagramme de log-probabilité a mis en évidence le caractère log-normal de ces deux variables (figs. 4 et 5). Le test de conformité du Khi-deux qui sert à évaluer les écarts entre les valeurs théoriques et empiriques sur des observations données montre que l'ajustement à la loi lognormale est satisfaisant au seuil de signification de $10 \%$ (tableau 3). La distribution des variables selon la loi log-normale permet d'envisager une modélisation géostatistique future. En effet, les valeurs log sont mieux structurées dans les variogrammes que les valeurs brutes. Il est donc recommandé d'utiliser celles-ci dans les études de modélisation.

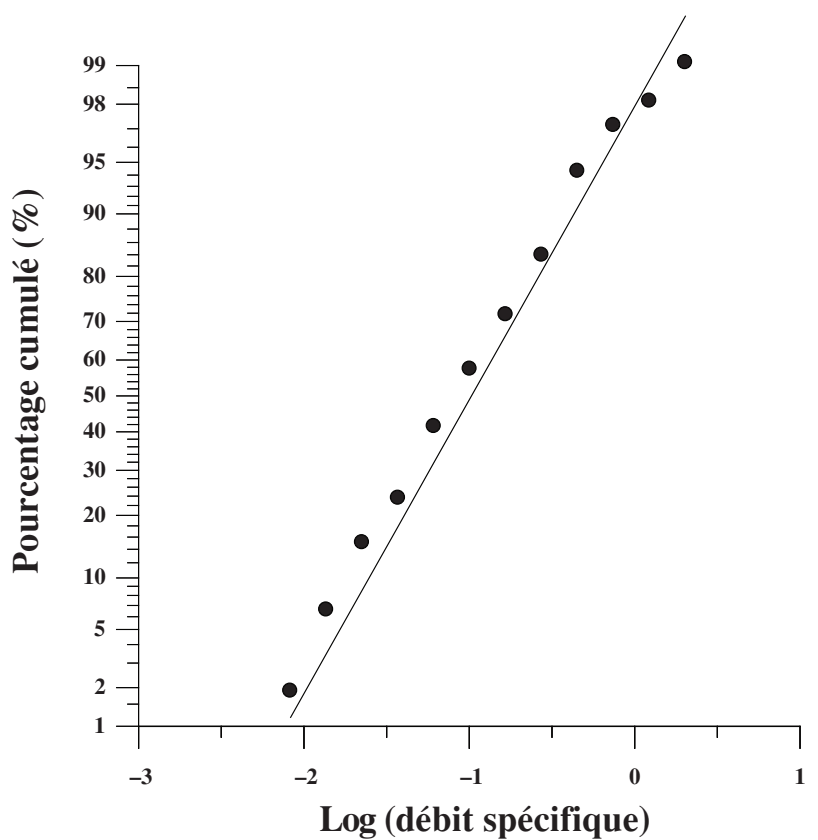

Fig. 5.-Fréquence de distribution du débit spécifique $\left(\mathrm{m}^{2} / \mathrm{h}\right)$ dans un diagramme log-normal.

\section{Relation empirique entre la transmissivité et le débit spécifique}

La représentation dans un graphique $\log T$ en fonction de $\log (Q / s)$ est donnée par la figure 6. On observe une faible dispersion des points. Le nuage de points obtenu présente une certaine tendance et les différents points s'alignent suivant une droite d'équation $7(\log T=1,188 \log (Q / s)-0,028)$ avec un intervalle de confiance de $95 \%$ qui traduit l'incertitude liée à l'estimation de la transmissivité. Cette équation peut également s'écrire sous la forme d'une loi puissance d'équation (8) avec un cœfficient de détermination $\mathrm{R}^{2}=0,82$.

$$
\log T=1,188 \log (Q / s)-0,028 \log T
$$

$\mathrm{Ou}$

$$
T=0,937(Q / s)^{1,188}
$$

Dans l'équation $T$ et $Q / s$ sont exprimés en $\mathrm{m}^{2} / \mathrm{h}$ et le débit spécifique n'a pas été corrigé des pertes de charges.

La valeur du coefficient de détermination signifie que $82 \%$ des valeurs de la transmissivité dans la zone d'étude sont liées aux débits spécifiques dans l'intervalle de confiance défini. On constate que le 


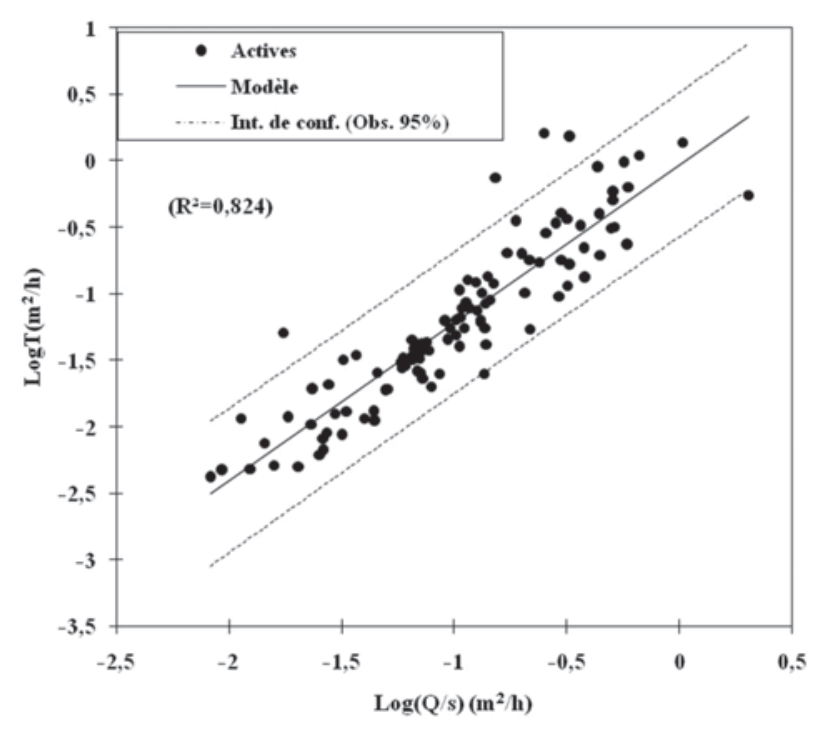

Fig. 6.-Relation entre $\log T$ et $\log (Q / s)$.

coefficient de détermination $R^{2}$ se situe dans la fourchette des différents coefficients relevés des relations citées précédemment. Par contre la pente 1,188 est plus élevée que celles rencontrées couramment. En somme, la relation définie pour les aquifères discontinus de la région des Lacs est nettement différente de celles existant dans la littérature.

\section{Discussion}

L'absence d'une corrélation significative entre la profondeur totale et le débit spécifique est due au fait qu'en zone de socle lors des campagnes d'hydraulique villageoise, la décision d'arrêt de foration n'est pas rationalisée (Kouadio et al., 2010). Certains forages sont précocement arrêtés dès l'obtention du débit escompté. Par contre, d'autres sont prolongés jusqu'au métré maximum défini dans les clause techniques du projet, surtout lorsque le résultat du sondage est négatif (Kouadio et al., 2010). De ce fait, les forages les plus profonds correspondent généralement au moins productifs. La profondeur d'un forage donné est donc sous l'influence du débit recherché et ne repose sur aucune considération technique et scientifique. Or, selon l'environnement géologique et hydrogéologique, il existe une profondeur au-delà de laquelle les chances de trouver un horizon aquifère s'amenuisent, notamment au sein des roches de socle altérés, du fait de la diminution de la fréquence puis de la disparition des fissures perméables (Dewandel et al., 2006). Par ailleurs, des études statistiques entre la productivité et la profondeur d'un puits ont montré que la probabilité de rencontrer des fractures ouvertes au delà d'une profondeur comprise entre 90-100 mètres est négligeable (Faillat, 1986). Selon Dewandel et al. (2006), l'essentiel de la perméabilité des aquifères de socle provient de la partie inferieure du profil d'altération, l'horizon fissuré stratiforme qui est situé sous les altérites meubles (lorsque celles-ci n'ont été érodées). Cependant, la présence de cet horizon ne garanti pas une bonne productivité de l'aquifère. En effet, les fractures hydrauliquement actives ont tendance à se fermer avec la profondeur. De même, elles peuvent se colmater si les arènes qui les recouvrent sont des argiles. On constate que les 60 premiers mètres de la roche non altérée sont les plus productifs. Ainsi, lorsque l'altération est peu développée, les venues d'eau semblent être très importantes. L'analyse statistique des données montre que la profondeur moyenne des arrivées d'eau est de 42,94 m et que les premières arrivées d'eau importantes se trouvent dans les 30 premiers mètres du socle fissuré dans la région des Lacs. Cette estimation est similaire à celle mise en évidence par Dewandel et al. (2006), dans les granites de l'Inde et à celle de Kouadio et al. (2010) dans le socle du Nord-Ouest de la Côte d'Ivoire. En effet, la plus grande partie de la fracturation hydrauliquement active se rencontre dans les $30 \mathrm{~m}$ de la roche saine dans tous les pays de socle. Selon Fahy (1980, 1981), les 30 premiers mètres de la roche saine sont les plus productifs lorsque celleci est fissurée. Gassita et al., 1987 abondent dans le même sens. La profondeur des venues d'eau est donc dépendante de l'état de fissuration ou de fracturation de l'aquifère d'où de la tectonique cassante. Dans ce cas, l'importance des débits rencontrés est liée à l'ouverture ou à la fermeture de ces fractures. La dynamique de ces fractures était considérée depuis les années 1970 sous le contrôle de la tectonique (Lachassagne \& Wyns, 2005). Cependant, depuis la fin des années 90, il est prouvé par plusieurs études menées sur le socle que cet aquifère doit sa perméabilité à l'existence d'un horizon fissuré stratiforme sous les altérites meubles d'épaisseur allant de 50 à $100 \mathrm{~m}$ (Cho et al., 2001; Lachassagne \& al., 2001). L'origine de cette fracturation résulte des contraintes engendrées par le gonflement des minéraux tels les phyllosilicates au cours du processus d'altération avec une fréquence des fissures qui décroît en profondeur (Wyns et al., 2004). Les para- 
mètres hydrauliques des aquifères sont donc plus ou moins en relation avec la géométrie des roches. Ainsi, les valeurs relativement élevées des transmissivités observées pourraient être dues à une densité de fracturation de ces roches. Mais l'existence d'une densité de fracturation élevée ne suffit pas à elle seule pour garantir de bonnes propriétés hydrauliques s'il n'existe pas une bonne connexion des réseaux de fractures. L'existence de joints subverticaux dans l'aquifère, contribue selon Lachassagne \& Wyns (2005), à la connexion de ce réseau de fractures. Seule une activité tectonique intense caractérisée par une réactivation d'anciennes fractures ou la mise en place de nouvelles fractures pourrait être à l'origine de ces propriétés hydrauliques (Faillat, 1986). Il semble également exister plusieurs indicateurs géologiques et hydrogéologiques qui indiquent une continuité et une homogénéité latérale du processus d'altération qui est à l'origine de la fracturation et qui pourraient agir sur la perméabilité de l'aquifère (Maréchal et al., 2004). En effet, l'altération joue un rôle primordial dans l'origine des fractures et influence par conséquent les paramètres hydrodynamiques des aquifères. La valeur moyenne de l'altération de cette étude est de 16,52 m. Elle est inférieure à la moyenne obtenue par Leblond (1984) dans la zone de Yamoussoukro qui est de $25 \mathrm{~m}$. Par ailleurs, la zone d'étude est dominée dans sa majeure partie par les granites sur lesquels l'épaisseur des altérites est faible comparée aux schistes qui sont plus tendres. En effet, sur les granitoïdes en Côte d'Ivoire, l'épaisseur des profils d'altération est comprise entre 50$60 \mathrm{~m}$. Par contre sur les schistes, elle est en moyenne de $80 \mathrm{~m}$ et souvent elle atteint $100 \mathrm{~m}$ dans l'ancienne Boucle de Cacao (Fahy, 1980, 1981). Tout ceci confirme le rôle que joue la nature pétrographique du réservoir dans l'évolution des propriétés hydrauliques des aquifères. Dans les granites, seules quelques fractures subhorizontales présentent une perméabilité suffisante pour permettre des «venues d'eau» significatives. La perméabilité est voisine de $10^{-4} \mathrm{~m} / \mathrm{s}$ et l'extension latérale est comprise entre 5 et 40 m environ (Lachassagne \& Wyns, 2005).

Le débit spécifique permet d'évaluer la qualité d'un ouvrage car il prend en compte la productivité du couple «nappe-ouvrage» (Sinan et al., 2003). Selon Biémi (1992), l'on peut à partir des valeurs des débits spécifiques avoir une idée de l'ampleur d'un programme de campagne d'hydraulique dans une région ainsi que les techniques employées pour l'implantation des forages. En effet, lors des cam- pagnes d'hydraulique villageoise comme c'est le cas pour les données de cette étude, la recherche de gros débits n'est pas l'objectif premier. Un forage est déclaré positif si, le débit atteint est supérieur ou égal à $1 \mathrm{~m}^{3} / \mathrm{h}$ quelle qu'en soit la profondeur du forage. La recherche d'une fracture très productive n'est donc pas une priorité. C'est sans doute ce qui pourrait expliquer les faibles valeurs de débits spécifiques estimées dans la zone d'étude. De même, un mauvais développement du puits pourrait également affecter les valeurs de débit spécifique. La distribution des fréquences des valeurs transformées $(\log T$ et $\log Q / s)$ suivant la loi lognormale est en accord avec les observations faites sur les aquifères hétérogènes dans la littérature (Delhomme, 1979; Jalludin \& Razack, 2004; Razack \& Lasm, 2006; Yidana et al., 2007). Cette distribution est le reflet des propriétés statistiques des paramètres de la facturation dont la plupart obéit à cette loi (Lasm, 2000). Ce résultat serait lié à l'hétérogénéité du milieu fracturé (Lasm, 2000) et peut donc s'avérer fort intéressant. En effet, la structuration d'un champ de transmissivités apparaît beaucoup plus forte si l'on étudie le logarithme de $T$ plutôt que sa valeur brute (De Marsily, 1994; Painter et al., 2007). Selon Painter et al. (2007), les logarithmes transformés de $T$ sont généralement utilisés pour le calcul des semi-variogrammes ou corrélogrammes. Des résultats analogues ont été trouvés par plusieurs auteurs (Delhomme, 1979; Jalludin \& Razack, 2004; Razack \& Lasm, 2006, Yidana et al., 2007). L'estimation de la transmissivité à partir du débit spécifique peut donc se faire avec une marge d'erreur acceptable de $95 \%$. En effet, la relation qui unit la transmissivité au débit spécifique indique que ce dernier est l'expression de la géométrie des fractures et de leur connexion avec l'ouvrage (Lasm, 2000). Le débit spécifique est probablement fonction du nombre de fractures interceptées par le puits et de ce fait il se trouve influencé par la profondeur d'équipement et le développement du puits. La relation déterminée entre $T$ et $Q / s$ dans cette étude diffère considérablement de celles obtenues par Razack \& Lasm (2006), Acheampong \& Hess (1998) et Yidana et al. (2007). Toutes choses qui confirment le fait que les relations entre $T$ et $Q / s$ varient d'un site à un autre et sont donc spécifiques au site d'étude. Le paramètre de régression $A_{2}$ obtenu dans cette étude est de 0,936 . Il se situe dans l'intervalle 0,9 à 1,52 des valeurs théoriques de Thomasson (1960) cité par Rotzoll \& El-Kadi (2008). En comparant la pente $(D)$ de régression de la rela- 
tion puissance de la région des Lacs $(D=1,188)$ avec celles des aquifères discontinus du bassin de la Volta au Ghana $(D=1,075)$ (Yidana et al., 2007) et des aquifères cristallin et métamorphique fracturés de la région de Man-Danané, à l'ouest de la Côte d'Ivoire $(D=1,30)$ (Razack \& Lasm, 2006), on constate que ces pentes sont très proches. Ces résultats montrent que ces aquifères pourraient avoir des similitudes dans leur comportement. Par contre, la pente $D$ est supérieure à celle des aquifères volcaniques de la République de Djibouti (Jalludin \& Razack, 2004), où le débit spécifique a été corrigé des pertes de charges $(D=0,938)$. Elle est également plus grande que celle des aquifères volcaniques de l'île de Jeju en Corée du Sud $(D=0,89)$ (Hamm et al. (2005)). De faibles pentes ou des pentes différentes de l'unité pourraient être dues à la corrélation qui existe entre $Q / s$ et le rendement du puits (Christensen, 1995). Il a été également montré par Razack \& Huntel (1991) et Jalludin \& Razack (2004) que les pertes de charge dans le puits affectent la relation entre $Q / s$ et $T$. Cependant, il semble difficile d'expliquer la différence entre la pente des aquifères cristallins et cristallophylliens de l'ouest de la Côte d'Ivoire et celle des aquifères du centre. Hamm et al. (2005) et Razack \& Lasm (2006) en comparant différentes relations empiriques de différents systèmes aquifères ont conclu que la différence entre les constantes de régression est probablement due à la géologie, au développement du puits, aux caractéristiques hydrauliques des aquifères, à la profondeur d'équipement, à la durée de pompage $(t)$, à la pénétration partielle du puits, au coefficient d'emmagasinement $(S)$, et les modèles utilisées pour déterminer les paramètres des aquifères. Selon Yidana et al. (2007) les propriétés hydrauliques des aquifères résulteraient d'une fracturation secondaire induite par des évènements structuraux régionaux dans les roches et non pas seulement d'une porosité primaire. En milieu discontinu, les réseaux fissurés conducteurs sont de géométrie complexe suite à une histoire structurale polyphasée ce qui leur confère des propriétés hydrauliques très variables. La transmissivité tout comme le débit spécifique est affecté par la perte de charge dans le puits. Ainsi, si les pertes de charge sont élevées, la relation entre $T$ et $Q / s$ se trouve très biaisée (Hamm et al., 2005). Par ailleurs, une correction du débit spécifique des pertes de charge peut permettre d'obtenir une estimation précise de la transmissivité. Dans cette étude, le débit spécifique n'a pas été corrigé des pertes de charge. De ce fait, la relation entre $T$ et $Q / s$ pourrait se trouver affectée à cause de l'effet dû à ces pertes de charge. D'autre part, une estimation indirecte de ces pertes de charge est incertaine et pourrait causer des estimations imprécises de la transmissivité (Hamm et al., 2005). Pour Meier et al. (1999), les paramètres de l'équation de régression obtenus entre $T$ et $Q / s$ sont affectés par l'hétérogénéité de l'aquifère, la période d'analyse et les pertes de charges dues à la turbulence au niveau du puits. Ainsi, chacun de ces facteurs pourrait donc affecter les paramètres de l'équation de régression. La compréhension et la prise en compte de ces facteurs permettraient d'expliquer la variabilité de ces différentes relations empiriques et aussi d'améliorer la relation trouvée dans cette étude. L'estimation de la transmissivité par cette relation empirique déterminée est fort utile pour palier le manque de données dans les études de modélisation.

\section{Conclusion}

L'étude de 105 forages dans la région des Lacs dans le centre de la Côte d'Ivoire a permis d'obtenir plusieurs résultats. La profondeur totale des forages varie de 49,50 à $99 \mathrm{~m}$ avec une moyenne de $69 \mathrm{~m}$. Cette profondeur fixée lors des études de bureau est assujettie à l'obtention d'un débit appelé débit positif. L'épaisseur des altérites est comprise entre 1,90 et $63,10 \mathrm{~m}$ avec une moyenne de $16,52 \mathrm{~m}$. Les arrivées d'eau correspondant aux profondeurs hydrauliquement actives ont une moyenne de $42,94 \mathrm{~m}$ avec des extrêmes compris entre 10 et $87 \mathrm{~m}$. Le débit des forages est compris entre 0,10 et $10,80 \mathrm{~m}^{3} / \mathrm{s}$ pour une moyenne de $2,32 \mathrm{~m}^{3} / \mathrm{s}$. Les valeurs de transmissivité obtenues par la méthode de remontée de Jacob sont comprises entre $1,15 \cdot 10^{-6}$ et $4,48 \cdot 10^{-4} \mathrm{~m}^{2} / \mathrm{s}$ et s'étendent sur trois ordres de grandeur, avec une moyenne de $4,95 \cdot 10^{-5} \mathrm{~m}^{2} / \mathrm{s}$. Les valeurs de débit spécifique estimées varient entre $2,30 \cdot 10^{-6}$ et $5,63 \cdot 10^{-4} \mathrm{~m}^{2} / \mathrm{s}$ avec une moyenne de $5,19 \cdot 10^{-5} \mathrm{~m}^{2} / \mathrm{s}$. Tout comme les transmissivités, les débits spécifiques s'étendent sur trois ordres de grandeur avec des coefficients de variation (CV) supérieurs à $100 \%$ ce qui montre la forte hétérogénéité structurale de l'aquifère. Aucune corrélation n'a été déterminée entre la profondeur totale des forages et les débits spécifiques et aussi entre l'épaisseur d'altération et la profondeur hydrauliquement active. Cependant, les forages les moins profonds présentent les débits spécifiques les plus élevés. Le maxi- 
mum des venues d'eau se rencontrent dans les 60 premiers mètres forés dans la roche non altérée. De plus, les profondeurs hydrauliquement actives sont importantes lorsque l'épaisseur d'altération est comprise entre 0 et $30 \mathrm{~m}$. L'ajustement statistique des valeurs transformées de $T$ et de $Q / s$ suivant la loi log-normale a été satisfaisant au seuil de $10 \%$ ce qui offre une perspective pour une étude de modélisation de ces aquifères. La relation empirique établie entre la transmissivité et le débit spécifique non corrigé $T=0,937(Q / s)^{1,188}$ présente des paramètres différents de celles de certaines études effectuées dans des contextes présentant des paramètres hydrogéologiques différents. Les pentes de régression des relations trouvées dans le bassin de la Volta au Ghana et à l'ouest de la Côte d'Ivoire sont sensiblement identiques et pourraient être dues à des paramètres hydrogéologiques similaires. Comme relevé par des travaux antérieurs, la perte de charge au niveau du puits semble être l'un des principaux facteurs responsable des valeurs élevées ou faibles de la pente de régression dans ces différentes équations. De même, la géologie, le modèle d'aquifère étudié, le développement du puits et le temps de pompage peuvent aussi contribuer à sous-estimer ou surestimer la transmissivité. De ce fait, cette relation pourrait être améliorée en corrigeant le débit spécifique des pertes de charge.

\section{REMERCIEMENTS}

Les auteurs remercient le Directeur de l'Hydraulique Humaine de Côte d'Ivoire ainsi que tous ses collaborateurs et notamment le Directeur Territorial de l'Hydraulique de la Région des Lacs pour leur disponibilité lors des différentes sorties effectuées sur le terrain.

\section{Références}

Acheampong, S.Y. \& Hess, J.W. (1998). Hydrogeological and hydrochemical framework of the shallow groundwater system in the southern Voltaian Sedimentary Basin, Ghana. Hydrogeology Journal, 6: 527-537. doi:10.1007/s100400050173

Biémi, J. (1992). Contribution à l'étude géologique, hydrogéologique et par télédétection des bassins versants subsaheliens du socle précambrien d'Afrique de l'Ouest: Hydrostructurale hydrodynamique, hydrochimie et isotopie des aquifères discontinus de sillons et aire granitique de la Haute Marahoué (Côte d'Ivoire). $\mathrm{PhD}$ Thesis (Thèse de Doctorat ès Sc. Nat.), Université de Cocody, Abidjan, Côte d'Ivoire, 479 pp.
CEFIGRE (1990). L'Hydrogéologie de l'Afrique de l'Ouest. Synthèse des connaissances sur l'hydrogéologie du socle cristallin et cristallophyllien et du sédimentaire ancien de l'Afrique de l'Ouest. Colloque Maîtrise de l'eau $2^{\mathrm{e}}$ ed., 147 pp.

Chapuis, R.P. (2007). Guide des essais de pompage et leurs interprétations. Bibliothèque et archives nationales du Québec, Gouvernement du Québec, Canada, $155 \mathrm{pp}$.

Cho, M.; Choi, Y.; Ha, K.; Kee, W.; Lachassagne, P. \& Wyns, R. (2003). Relationship between the permeability of hard rock aquifers and their weathering, from geological and hydrogeological observations in South Korea. International Association of Hydrogeologists Conference on «Groundwater in fractured rocks», Prague.

Christensen, S. (1995). Prediction of log-transmissivity 1: using specific capacity. Nordic Hydrology, 26: 1-20.

Delhomme, J.P. (1979). Spatial variability and uncertainty in groundwater flow parameters: a geostatistical approach. Water Resources Research, 15: 269-280. doi:10.1029/WR015i002p00269

De Marsily, G. (1994). Hydrogéologie: Comprendre et estimer les écoulements souterrains et le transport des polluants. Ecole des mines de Paris, 242 pp.

Detay, M. (1987). Méthodologie d'implantation des ouvrages, type hydraulique villageoise par étude de terrain et photo-interprétation. Session Internationale de Formation «Evaluation, aménagement et gestion des ressources en eau», Résume de cours Doc CEFIGRE, 20 pp.

Detay, M.; Poyet, P.; Emsellem, Y.; Bernadi, A. \& Aubrac, G. (1989). Influence du développement du réservoir capacitif d'altérites et de son état de saturation sur les caractéristiques hydrodynamiques des forages en zones de socle cristallin. Comptes Rendus de la Académie des Sciences Série II, 309: 429-436.

Detay, M. (1993). Le forage d'eau: Réalisation, entretien, réhabilitation. Editions Masson, Paris, 379 pp.

Dewandel, B.; Lachassagne, P.; Wyns, R.; Maréchal, J.C. \& Krishnamurthy, N.S. (2006). A generalized 3-D geological and hydrogeological conceptual model of granite aquifers controlled by single or multiphase weathering. Journal of Hydrology, 330: 260-284. doi:10.1016/j.jhydrol.2006.03.026

DHH (2001). Hydraulique Humaine en Cote d'Ivoire. Ministère des Infrastructures Economiques, Direction de l'Hydraulique Humaine, Abidjan, 66 pp.

Engalenc, M. (1978). Méthodes d'études et de recherche de l'eau souterraine des roches cristallines. Publication du Comité Inter-Africain d'Etudes Hydrauliques 1, Ouagadougou, Burkina Faso, 250 pp.

Fabre, R. (1985). Les effets tectoniques des décrochements N-S senestre dans les formations volcaniques birrmiennes de la région du Yaouré centre de la Côte d'ivoire. Comptes Rendus de l'Académie des Sciences, série II, 19: 955-960.

Faillat, J.P. (1986). Hétérogénéité et effet d'échelle dans les aquifères fissurés. Approche par pompage d'essai sur station expérimentale (Afrique de l'Ouest). Hydrogéologie, 1: 65-76. 
Faillat, J.P. (1987). Aquifères fissurés en zone tropicale humide: structure, hydrodynamique et hydrochimie (Afrique de l'Ouest). Hydrogéologie, 2: 109-112.

Fahy, J.C. (1980). Exploitation et entretien des points d' eau villageois en Côte d'Ivoire. Resumes du 26th international geological congress, Paris, France, 26: 1093.

Fahy, J.C. (1981). Exploitation et entretien des points d'eau villageois en Côte d'Ivoire. Bulletin du Bureau de Recherches Geologiques et Minieres, 4: 339-341.

Hamm, S.Y.; Cheong, J.Y.; Jang, S.; Jung, C.Y. \& Kim, B.S. (2005). Relationship between transmissivity and specific capacity in the volcanic aquifers of Jeju Island, Korea. Journal of Hydrology, 310: 111-121. doi:10.1016/j.jhydrol.2004.12.006

Gassita, S.; Gageonnet, M. \& Solages, S. (1987). Synthèse des résultats du premier programme d'hydraulique rurale exécutée en République du Gabon. Hydrogéologie, 2: 113-126.

Gingerich, S.B. (1999). Estimating transmissivity and storage properties from aquifer tests in the southern Lihue basin, Kauai, Hawaii. U.S. Geological Survey, WaterResources Investigations Report 99-4066, 33 pp.

INS (2001). Recensement général de la population et de l'Habitat 1998. Données socio-démographiques et économiques: Région des Lacs. Institut National de la Statistique, Bureau Technique du Recensement, Abidjan, Côte d'Ivoire.

Jacob, C.E. (1947). Draw-down test to determine effective radius of artesian well. Transactions, American Society of Civil Engineers, 112: 1047-1070.

Jalludin, M. \& Razack, M. (2004). Assessment of hydraulic properties of sedimentary and volcanic aquifer systems under arid conditions in the Republic of Djibouti (Horn of Africa). Hydrogeology Journal, 12: 159-170. doi:10.1007/s100400030312-2

Kouadio, K.E.; Soro, N. \& Savané, I. (2010). Stratégie d'optimisation de la profondeur des forages en contexte de socle: application à la région du Denguélé, NordOuest de la Côte d'Ivoire. Revue des Sciences de l'Eau, 23: 1-15.

Kouassi, A.M.; Kouame, K.F.; Saley, B.M. \& Koffi, Y.B. (2007). Identification of tendencies with in the rainfall-runoff relation and refill of the tablecloths in a context of hydroclimatic variability in the catchment area of N'zi (Bandama) in Ivory Coast. European Journal of Scientific Research, 16: 412-425.

Kruseman, G.P.; De Ridder, N.A. \& Meilhac, A. (1974). Interprétation et discussion des pompages d'essais. International Institute for Land Reclamation and Improvement, Wageningen, The Netherlands, 210 pp.

Kruseman, G.P. \& De Ridder, N.A. (1991). Analysis and evaluation of pumping test data. International Institute for Land Reclamation and Improvement, Wageningen, The Netherlands, $341 \mathrm{pp}$.

Lachassagne, P.; Ahmed, S.; Golaz, C.; Maréchal, J.C.; Thiery, D.; Touchard, F. \& Wyns, R.A. (2001). Methodology for the mathematical modelling of hard-rock aquifers at catchment scale based on the geological structure and the hydrogeological functioning of the aquifer. In: New approaches characterizing groundwa- ter flow (Seiler, K.-P. \& Wohnlich, S., eds.). XXXI IAH Congress «New approaches characterizing groundwater flow», A.A. Balkema Publishers Munich, Germany, 367-370.

Lachassagne, P.; Maréchal, J.-C; Dewandel, B.; Gandolfi, J.M.; Krishnamurthy, N.S.; Surbrahmanyam, K. \& Wyns, R.A. (2005). Nouveaux outils et méthodes pour gérer et protéger la ressource en en eau souterraine des régions de socle. Hydrosciences, 150: 55-57.

Lachassagne P.; Wyns, R.; Bérard, P.; Bruel, T.; Chéry, L.; Coutand, T.; Desprats, J.-F. \& Le Strat, P. (2001). Exploitation of high-yield in hard-rock aquifers: Downscaling methodology combining GIS and multicriteria analysis to delineate field prospecting zones. Ground Water, 39: 568-581. doi:10.1111/j.1745-6584.2001.tb02345.x

Lachassagne, P. \& Wyns, R. (2005). Aquifères de socle: nouveaux concepts Application à la prospection et la gestion de la ressource en eau. Géosciences, 2: 32-37.

Lasm, T. (2000). Hydrogéologie des réservoirs fracturés de socle: analyses statistiques et géostatistique de la fracturation et des propriétés hydrauliques, application à la région des montagnes de Côte d'Ivoire (Domaine Archéen). PhD Thesis, Université de Poitiers, France, 220 pp.

Leblond, P. (1984). Contribution aux études hydrogéologiques en Côte d'Ivoire. Région de Yamoussoukro (Station expérimentale de l'ENSTP). Thèse de 3è cycle de l'Université de Bordeaux 1, 150 pp.

Maréchal, J.-C.; Dewandel, B.; Ahmed, S.; Galeazzi L. \& Zaidi, F.K. (2006). Combined estimation of specific yield and natural recharge in semi arid groundwater basin irrigated agriculture. Journal of Hydrology, 329: 281-293. doi:10.1016/j.jhydrol.2006.02.022

Maréchal, J.-C.; Dewandel, B. \& Subrahmanyam, K. (2004). Use of hydraulic tests at different scales to characterize fracture network properties in the weatheredfractured layer of a hard rock aquifer. Water Resources Research, 40: W1150801-W1150817.

Mbonu, M. (1991). Hydrogéologie, hydrochimie et Géochimie isotopique des aquifères de socle du Plateau de Jos et des plaines environnantes (Nigeria). PhD Thesis. Université d'Avignon et des Pays de Vaucluse. France, $171 \mathrm{pp}$.

Meier, P.M.; Carrera, J. \& Sanchez-Villa, X. (1999). A numerical study on the relationship between transmissivity and specific capacutiy in heterogenous aquifers. Ground Water, 37: 611-617. doi:10.1111/j.1745-6584.1999.tb01149.x

N'guessan, A. (1985). Contribution à l'hydrogéologie de la région Centrale de la Côte d'Ivoire et analyse statistique des résultats de forage. Thèse de 3è cycle, Université de Franche-Comté, Besançon, France, 154 pp.

Painter, S.L.; Woodbury, A.D. \& Jiang, Y. (2007). Transmissivity estimation for highly heterogeneous aquifers: comparison of three methods applied to the Edwards Aquifer, Texas, USA. Hydrogeology Journal, 15: 315-331. doi:10.1007/s10040006-0071-y

Razack, M. \& Lasm, T. (2006). Geostatistical estimation of the transmissivity in a highly fractured metamorphic 
and crystalline aquifer (Man-Danane Region, Western Ivory Coast). Journal of Hydrology, 325: 164-178. doi:10.1016/j.jhydrol.2005.10.014

Razack, M. \& Huntel, D. (1991). Assessing transmissivity from specific capacity in a large and heterogeneous aquifer. Ground Water, 29: 856-861. doi:10.1111/j.1745-6584.1991.tb00572.x

Rotzoll, K. \& El-Kadi, A.I. (2008). Estimating hydraulic conductivity from specific capacity for Hawaii aquifers, USA. Hydrogeology Journal, 16: 969-979. doi:10.1007/s10040-007-0271-0

Savadogo, A.N. (1984). Géologie et Hydrogéologie du socle cristallin de Haute -Volta. Etude régionale du bassin versant de la Sissili. $\mathrm{PhD}$ Thesis, Université Scientifique et Médicale de Grenoble, France, 351 pp.

Sinan, M.; Maslouhi, R. \& Razack, M. (2003). Utilisation des SIG pour a caractérisation de la vulnérabilité et de la sensibilité à la pollution des nappes d'eau souterraine. Application à la nappe du Haouz de Marrakech, Maroc. $2^{\text {nd }}$ FIG Regional Conference, TS11 Management of Water Resources, Marrakech, Morocco, 15 pp.

Soule de Lafont, D. (1956). Le précambrien moyen et supérieur de Bondoukou (Côte d' Ivoire). Bulletin de la Direction Fédérale des Mines et de la Géologie, 18: 1-174.

Soro, N.; Soro, G.; Ahoussi, K.E.; Saley, M.B.; Lasm, T. \& Biemi J. (2007). Caractérisation spatio-temporelle des précipitations dans le $« \mathrm{~V} »$ Baoulé (Centre de la Côte d'Ivoire) au cours de la période 1966-2000. Journal Africain de Communication Scientifique et Technologique, 2: 89-108.

Soro, N. (2002). Hydrochimie et géochimie isotopique des eaux souterraines du degré carré de Grand-Lahou et ses environs (Sud Ouest de la Côte d'Ivoire). Implication hydrologique et hydrogéologique. $\mathrm{PhD}$ Thesis, Université de Cocody, Côte d'Ivoire, 272 pp.

Theis, C.V. (1935). The relation between the lowering of the piezometric surface and the rate and duration on discharge of the well using ground-water storage. Transactions of the American Geophysical Union, 16: 519-524.

Yacé, I. (1976). Le volcanisme éburnéen dans les parties centrales et méridionales de la chaîne précambrienne de Fétékro en Côte d' Ivoire. $\mathrm{PhD}$ Thesis, Université d'Abidjan, Côte d'Ivoire, 373 pp.

Yacé, I. (2002). Initiation à la géologie. L'exemple de la Côte d'Ivoire et de l'Afrique de l'Ouest. Pétrologie, Géologie régionale. Centre d'edition et de diffusion africaines, Societe pour le Developpement Minier de la Côte D'Ivoire, 183 pp.

Yao, D.B. (1998). Lithostratigraphie et pétrologie des formations birrimiennes de Toumodi-Fettekro (Côte d'Ivoire): Implications pour l'évolution crustale du paléoprotérozoïque du craton ouest africain. $\mathrm{PhD}$ Thesis, Université d'Orléans. France, 191 pp.

Yidana, S.M.; Ophori, D. \& Banoeng-Yakubo, B. (2007). Hydrogeological and hydrochemical characterization of the Voltaian Basin: the Afram Plains area, Ghana. Environmental Geology, 54: 1751-1762.

Wyns, R.; Baltassat, J.M.; Lachassagne, P.; Legtchenko, A. \& Vairon, J. (2004). Application of proton magnetic resonance soundings to groundwater reserves mapping in weathered basement rocks (Brittany, France). Bulletin de la Société Géologique de France, 175: 21-34. doi:10.2113/175.1.21

Wyns, R.; Gourry, J.C.; Baltassat, J.M. \& Lebert F. (1999). Caractérisation multiparamétrés des horizons de subsurfaces $(0-100 \mathrm{~m})$ en contexte de socle altéré.

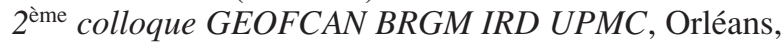
France, 105-110.

Recibido el 6 de noviembre de 2009 Aceptado el 9 de julio de 2010 Publicado online el 9 de diciembre de 2010 